\title{
Respuesta de un harnero vibratorio ante la pérdida de rigidez en los apoyos
}

\author{
Response of a vibrating screen when it loses stiffness in the supports \\ Manuel Moncada $^{1 *} \quad$ Cristian G. Rodríguez ${ }^{1}$ \\ Recibido 11 de enero de 2016, aceptado 13 de abril de 2016 \\ Received: January 11, 2016 Accepted: April 13, 2016
}

\begin{abstract}
RESUMEN
Los harneros vibratorios son máquinas críticas en la industria minera. La correcta operación del harnero se consigue con un movimiento adecuado, definido principalmente por su inclinación y amplitud vibratoria [1-2]. Los modelos dinámicos disponibles en la literatura no permiten simular condiciones en que es significativa la variación dinámica del ángulo de inclinación, como en la partida, detención y operación con pérdida de rigidez en algún apoyo, pues estos modelos linealizan el movimiento angular [3-4]. En el presente trabajo se plantea un modelo dinámico no lineal de tres grados de libertad de un harnero vibratorio mostrando su desarrollo, solución y simulaciones numéricas. Las simulaciones entregan cuánto cambia la dirección de cada órbita y magnitud de la frecuencia natural, con respecto a la rigidez de los apoyos. Con esto es posible evaluar el estado de los resortes para mantener las condiciones de diseño que aseguren la alta eficiencia requerida y detectar los cambios de estos para aumentar el tiempo medio de intervención por el reemplazo de los resortes.
\end{abstract}

Palabras clave: Modelo dinámico, no lineal, harnero vibratorio, pérdida de rigidez.

\begin{abstract}
Vibrating screens are critical machines in the mining industry. The screen movement is mainly defined by its inclination and vibration amplitude. A proper movement allows correct operation of the screen [1-2]. Available models cannot simulate motion with high angular displacement, as a startup, shutdown and under loss of stiffness in supporting positions, because these models linearize angular displacement [3-4]. In this article a 2D 3DOF nonlinear model is proposed, presenting its development, solution and numerical simulations. Simulations deliver how much the direction of each orbit and magnitude of the natural frequency change, with respect to the supports's stiffness. This makes it possible to evaluate the state of the springs to keep the design conditions to ensure high efficiency and detect changes required of them to increase the mean operation time for replacement of the springs.
\end{abstract}

Keywords: Dynamic model, nonlinear, vibrating screen, loss of stiffness.

\section{INTRODUCCIÓN}

Un harnero es una máquina utilizada en la industria, principalmente minera, para la clasificación de componentes sólidos en relación a su tamaño, con el objetivo de separar los productos finos de los gruesos. Contiene una o más mallas con múltiples aperturas de dimensiones uniformes, que actúan como un calibre pasa/no pasa, obteniendo un producto final con tamaño más equilibrado que el original.

El rendimiento de esta máquina es medido por la capacidad de separar el material correctamente, que

\footnotetext{
1 Departamento de Ingeniería Mecánica. Universidad de Concepción. Edmundo Larenas 219. Concepción, Chile. E-mail: manuelmoncada@udec.cl; crrodrig@udec.cl

* Autor de correspondencia
} 
se puede calcular por la fracción de material obtenida en cada salida [5]. La predicción numérica de esta magnitud se obtiene mediante modelos empíricos, como el de Karra [6] o por elementos discretos, como lo estudian en [1-2]. En ambos casos se evidencia que cambios en la inclinación, amplitud y aceleración vibratoria, afectan significativamente en su rendimiento.

Para conocer el movimiento del harnero vibratorio como cuerpo rígido se emplea la modelación dinámica. En la literatura existen dos modelos: el de He y Liu [3], y el de Liu [4]. El primero es lineal (linealizan la rotación $\theta$ ) de 3 grados de libertad, donde la excitación del sistema es circular y no está ubicado en el centro de masa. Para su resolución aprovechan la linealidad del sistema y expresan sus soluciones como sinusoidales. Al resolver sus ecuaciones con parámetros equivalentes, no se obtienen los mismos resultados, debido a la definición del ángulo de inclinación, que ellos definen como declinación y por la solución de $\theta$, que la amplitud obtenida no es la correcta. El segundo es un modelo lineal en 3D de tres grados de libertad, que fue desarrollado con las ecuaciones de Lagrange. Sus resultados son simulaciones del harnero cuando sus resortes pierden rigidez (como se hace en 3.2), pero no dejan en claro cuál es el comportamiento ni obtienen conclusiones al respecto. Ambos modelos solo son capaces de estudiar el estado estacionario, por tanto, la partida y detención no pueden ser estudiados, y por ser lineales, no pueden conocer la respuesta frente a altas rotaciones.

\section{MODELO NO LINEAL}

La linealización de los modelos comentados en la introducción se percibe en las rotaciones pues aproximan $\operatorname{sen} \theta \approx \theta$ y $\cos \theta \approx 1$, asumiendo que estos son bajos. En la práctica esto es cierto bajo condiciones nominales de operación (como se observará en 3.1), no obstante, existen situaciones en que el desplazamiento angular del harnero es alto, como la partida, detención y cuando los apoyos pierden rigidez, por ello la motivación es formular un modelo dinámico que pueda predecir el comportamiento de un harnero vibratorio cuando este está sometido a rotaciones significativas y, por ende, no linealizar en el planteamiento de las ecuaciones esos términos.
Se propone un modelo de un harnero de tres grados de libertad en el plano como el que se muestra en su posición de equilibrio en la Figura 1. La fuerza de excitación del sistema es variable en el tiempo en magnitud, $F$ sen $(\Omega t)$, y constante en dirección, $\alpha$ con respecto a la horizontal del harnero, $\mathrm{y}$ a una distancia $p$ del centro de masa. La base y material de carga del harnero vibratorio se definen como un cuerpo rígido inclinado un ángulo $\beta$ y el centro de masa está a una altura $h$, esto supone que no existe movimiento relativo entre la estructura de la máquina y la carga. El sistema de aislación está ubicado en los puntos $A$ y $B$, compuesto por una rigidez y amortiguación horizontal y vertical, cada uno a una distancia $a$ y $b$ de la proyección del centro de masa.

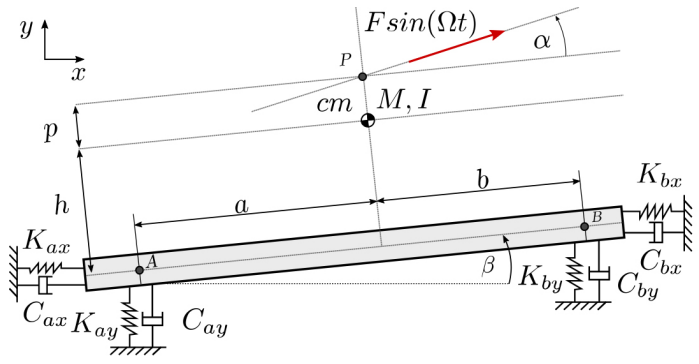

Figura 1. Modelo propuesto.

Usando las ecuaciones de Lagrange, se desarrollan las ecuaciones del movimiento para los tres grados de libertad $q_{1}=x, q_{2}=y$ y $q_{3}=\theta$. Conociendo que en este caso la energía cinética solo es función de $\dot{q}_{i}$, la potencial de $q_{i}$, y agregando la función de disipación de energía en un amortiguador viscoso entregada por Rayleigh, donde $F=\frac{1}{2} c \dot{q}$, la ecuación de Lagrange se escribe como:

$$
\frac{d}{d t}\left(\frac{\partial T}{\partial \dot{q}_{i}}\right)+\frac{\partial V}{\partial q_{i}}+\frac{\partial F}{\partial \dot{q}_{i}}=Q_{i}
$$

Para expresar estas ecuaciones es necesario dar un desplazamiento por cada grado de libertad, luego desarrollar las funciones de energía, los desplazamientos de los puntos $A$ y $B$ y la fuerza generalizada, para finalmente tener las ecuaciones del movimiento (2)(4). En las simulaciones en que se cambie la rigidez de los apoyos es necesario recalcular la inclinación del harnero, pues esta varía en condiciones de equilibrio, por lo que se plantean las ecuaciones que relacionan 
las fuerzas estáticas sobre el harnero y la geometría de los apoyos, semejante a lo desarrollado por [7].

$$
\begin{aligned}
& I \ddot{\theta}+2 \dot{\theta} \operatorname{sen} \theta \cos \theta \cdot[ \\
& C_{a y}(h \operatorname{sen} \beta-a \cos \beta)(h \cos \beta+a \operatorname{sen} \beta) \\
& +C_{b y}(h \operatorname{sen} \beta+b \cos \beta)(h \cos \beta-b \operatorname{sen} \beta) \\
& +C_{a x}(h \cos \beta+a \operatorname{sen} \beta)(-h \operatorname{sen} \beta+a \cos \beta) \\
& \left.+C_{b x}(-h \cos \beta+b \operatorname{sen} \beta)(h \operatorname{sen} \beta+b \cos \beta)\right] \\
& +\dot{\theta} \cos ^{2} \theta \cdot[ \\
& C_{a y}(h \operatorname{sen} \beta-a \cos \beta)^{2}+C_{b y}(h \operatorname{sen} \beta+b \cos \beta)^{2} \\
& \left.+C_{a x}(h \cos \beta+a \operatorname{sen} \beta)^{2}+C_{b x}(h \cos \beta-b \operatorname{sen} \beta)^{2}\right] \\
& +\dot{\theta} \operatorname{sen}^{2} \theta \cdot[ \\
& C_{a y}(h \cos \beta+a \operatorname{sen} \beta)^{2}+C_{b y}(-h \cos \beta+b \operatorname{sen} \beta)^{2} \\
& \left.+C_{a x}(h \operatorname{sen} \beta-a \cos \beta)^{2}+C_{b x}(h \operatorname{sen} \beta+b \cos \beta)^{2}\right] \\
& +\cos (\theta+\beta) \cdot\left[\dot{x} h\left(C_{a x}+C_{b x}\right)+\dot{y}\left(-a C_{a y}+b C_{b y}\right)\right. \\
& \left.x h\left(K_{a x}+K_{b x}\right)+y\left(-a K_{a y}+b K_{b y}\right)\right] \\
& +\operatorname{sen}(\theta+\beta) \cdot\left[\dot{x}\left(a C_{a x}-b C_{b x}\right)+\dot{y} h\left(C_{a y}+C_{b y}\right)\right. \\
& \left.x\left(a K_{a x}-b K_{b x}\right)+y h\left(K_{a y}+K_{b y}\right)\right] \\
& +(\cos 2 \theta-\cos \theta)\{ \\
& K_{a x}\left[a h\left(-\cos ^{2} \beta+\operatorname{sen}^{2} \beta\right)+\left(h^{2}-a^{2}\right) \operatorname{sen} \beta \cos \beta\right] \\
& +K_{a y}\left[a h\left(\cos ^{2} \beta-\operatorname{sen}^{2} \beta\right)+\left(-h^{2}+a^{2}\right) \operatorname{sen} \beta \cos \beta\right] \\
& +K_{b x}\left[b h\left(\cos ^{2} \beta-\operatorname{sen}^{2} \beta\right)+\left(h^{2}-b^{2}\right) \operatorname{sen} \beta \cos \beta\right] \\
& \left.+K_{b y}\left[b h\left(-\cos ^{2} \beta-\operatorname{sen}^{2} \beta\right)+\left(-h^{2}+b^{2}\right) \operatorname{sen} \beta \cos \beta\right]\right\} \\
& +\cos \theta \operatorname{sen} \theta \cdot\{ \\
& K_{a x}[4 a h \cos \beta \operatorname{sen} \beta \\
& \left.+\left(-h^{2}+a^{2}\right) \operatorname{sen}^{2} \beta+\left(h^{2}-a^{2}\right) \cos ^{2} \beta\right] \\
& -K_{a y}[4 a h \cos \beta \operatorname{sen} \beta \\
& \left.+\left(-h^{2}+a^{2}\right) \operatorname{sen}^{2} \beta+\left(h^{2}-a^{2}\right) \cos ^{2} \beta\right] \\
& -K_{b x}[4 b h \cos \beta \operatorname{sen} \beta \\
& \left.-\left(-h^{2}+b^{2}\right) \operatorname{sen}^{2} \beta-\left(h^{2}-b^{2}\right) \cos ^{2} \beta\right] \\
& +K_{b y}[4 b h \cos \beta \operatorname{sen} \beta \\
& \left.\left.-\left(-h^{2}+b^{2}\right) \operatorname{sen}^{2} \beta-\left(h^{2}-b^{2}\right) \cos ^{2} \beta\right]\right\} \\
& +\operatorname{sen} \theta \cdot\left[K_{a x}(a \cos \beta-h \operatorname{sen} \beta)^{2}\right. \\
& \begin{array}{l}
+K_{a y}(a \operatorname{sen} \beta+h \cos \beta)^{2} \\
+K_{b x}(h \operatorname{sen} \beta+b \cos \beta)^{2} \\
\left.+K_{b y}(h \cos \beta-b \operatorname{sen} \beta)^{2}\right] \\
=-F \operatorname{sen}(\Omega t) p \cos \alpha
\end{array}
\end{aligned}
$$$$
M \ddot{x}+[\dot{x}+\dot{\theta} h \cos (\theta+\beta)] \cdot\left(C_{a x}+C_{b x}\right)
$$$$
+\dot{\theta} \operatorname{sen}(\theta+\beta)\left(a C_{a x}-b C_{b x}\right)
$$$$
+[x+h \operatorname{sen}(\theta+\beta)] \cdot\left(K_{a x}+K_{b x}\right)
$$$$
+\cos (\theta+\beta)\left(-a K_{a x}+b K_{b x}\right)
$$$$
+K_{a x}(a \cos \beta-h \operatorname{sen} \beta)
$$$$
+K_{b x}(-h \operatorname{sen} \beta-b \cos \beta)
$$$$
=F \operatorname{sen}(\Omega t) \cos (\theta+\alpha+\beta)
$$

$$
\begin{aligned}
& M \ddot{y}+[\dot{y}+\dot{\theta} h \operatorname{sen}(\theta+\beta)] \cdot\left(C_{a y}+C_{b y}\right) \\
& +\dot{\theta} \cos (\theta+\beta)\left(-a C_{a y}+b C_{b y}\right) \\
& +[y-h \cos (\theta+\beta)] \cdot\left(K_{a y}+K_{b y}\right) \\
& +\operatorname{sen}(\theta+\beta)\left(-a K_{a y}+b K_{b y}\right) \\
& +K_{a y}(h \cos \beta+a \operatorname{sen} \beta) \\
& +K_{b y}(h \cos \beta-b \operatorname{sen} \beta) \\
& =F \operatorname{sen}(\Omega t) \operatorname{sen}(\theta+\alpha+\beta)
\end{aligned}
$$

\section{Modelo lineal equivalente}

Para ver las diferencias o ventajas que tiene el modelo propuesto con uno lineal, se enuncian las ecuaciones lineales del movimiento del harnero, como el expuesto en [3], mostradas en (5). Además de ser lineal se diferencian en que: los grados de libertad $x$ e $y$ están desacoplados de los demás y no dependen de la geometría del harnero, que la fuerza desprecia el desplazamiento angular $\theta$ y que no se considera el movimiento real de los apoyos.

$$
\begin{gathered}
M \ddot{x}+\dot{x} \cdot\left(C_{a x}+C_{b x}\right) \\
+x \cdot\left(K_{a x}+K_{b x}\right) \\
=F \operatorname{sen}(\Omega t) \cos (\alpha+\beta) \\
M \ddot{y}+\dot{y} \cdot\left(C_{a y}+C_{b y}\right) \\
\quad+y \cdot\left(K_{a y}+K_{b y}\right) \\
=F \operatorname{sen}(\Omega t) \operatorname{sen}(\alpha+\beta)
\end{gathered}
$$

Ï̈

$$
\begin{array}{r}
-\dot{x}\left(a C_{a x}+b C_{b x}\right) \operatorname{sen} \beta+\dot{y}\left(a C_{a y}-b C_{b y}\right) \cos \beta \\
-x\left(a K_{a x}+b K_{b x}\right) \operatorname{sen} \beta+y\left(a K_{a y}-b K_{b y}\right) \cos \beta \\
{\left[-\dot{\theta}\left(a^{2} C_{a x}+b^{2} C_{b x}\right)+\theta\left(a^{2} K_{a y}+b^{2} K_{b y}\right)\right] \cos \beta} \\
=-F \operatorname{sen}(\Omega t) p \cos \alpha
\end{array}
$$

\section{SIMULACIONES}

Se pone a prueba el modelo propuesto simulando un harnero vibratorio en condiciones nominales de operación, cuando la rigidez de los apoyos disminuye discretamente, en una partida y se compara con un modelo lineal equivalente para ver las ventajas y/o diferencias. Las ecuaciones del movimiento (2)(4) son un sistema de ecuaciones diferenciales no lineales de segundo orden, como no existe solución analítica, se resuelven numéricamente con el método de Runge-Kutta de orden variable y paso múltiple [8]. Para efectuar las simulaciones se usan los datos presentados en la Tabla 1, entregando valores para el harnero con y sin carga. Los datos corresponden a un harnero usado en Chile en la minería del cobre. 
Tabla 1. Parámetros para la simulación, con y sin carga (las diferencias son en las filas 8 y 9 respectivamente).

\begin{tabular}{|c|c|c|c|c|}
\hline$F[\mathrm{~N}]$ & \multicolumn{2}{|c|}{$\Omega[\mathrm{rad} / \mathrm{s}]$} & $\alpha\left[^{\circ}\right]$ & $\beta\left[^{\circ}\right]$ \\
\hline 1.322 .071 & \multicolumn{2}{|l|}{98,96} & 135 & 4,8 \\
\hline$K_{a x}[\mathrm{~N} / \mathrm{m}]$ & \multicolumn{2}{|c|}{$K_{a v}[\mathrm{~N} / \mathrm{m}]$} & $K_{b x}[\mathrm{~N} / \mathrm{m}]$ & $K_{b y}[\mathrm{~N} / \mathrm{m}]$ \\
\hline 2.064 .000 & \multicolumn{2}{|c|}{4.992 .000} & 2.064 .000 & 4.992 .000 \\
\hline$C_{a x}[\mathrm{Ns} / \mathrm{m}]$ & \multicolumn{2}{|c|}{$C_{a y}[\mathrm{Ns} / \mathrm{m}]$} & $C_{b x}[\mathrm{Ns} / \mathrm{m}]$ & $C_{b y}[\mathrm{Ns} / \mathrm{m}]$ \\
\hline 100.000 & \multicolumn{2}{|c|}{50.000} & 100.000 & 50.000 \\
\hline$M[\mathrm{~kg}]$ & $I\left[\mathrm{kgm}^{2}\right]$ & $h[\mathrm{~m}]$ & $a[\mathrm{~m}]$ & $b[\mathrm{~m}] \quad p[\mathrm{~m}]$ \\
\hline 24.468 & 622.443 & 0,298 & 2,054 & 3,896 \\
\hline 48.497 & 1.156 .474 & 0 & 2,917 & 3,033 \\
\hline
\end{tabular}

\section{Simulación en condiciones nominales}

En la Figura 2 se presenta la respuesta estacionaria del harnero con y sin carga y en la Figura 3 (a) las órbitas del centro de masa para los dos casos, siendo el caso con carga el de un harnero en funcionamiento.

Al comparar las respuestas estacionarias se destaca principalmente un cambio de amplitud, por la diferencia de masa con que el sistema es simulado, $y$ un desfase entre las respuestas en $x$ e $y$, como

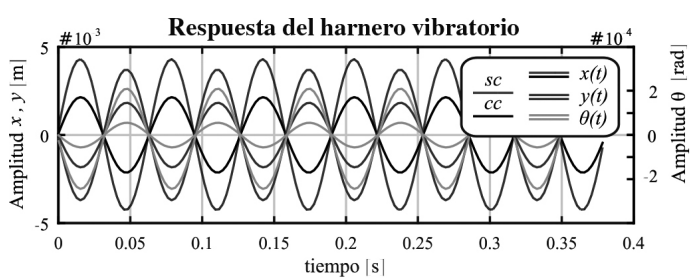

Figura 2. Respuesta estacionaria vertical, horizontal y angular del harnero (sc: sin carga, cc: con carga).

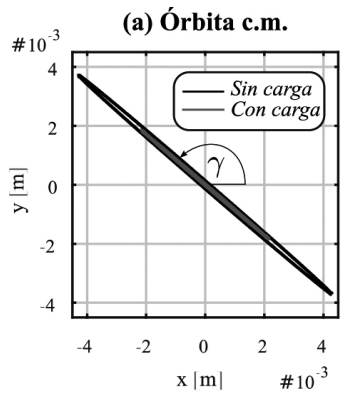

(b) Espectro de y(t) en $\mathbf{A}$

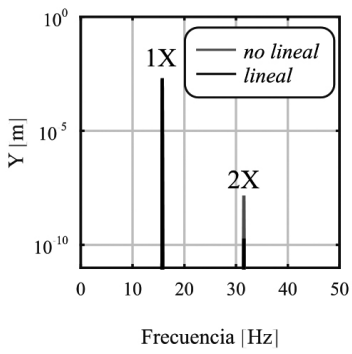

Figura 3. (a) Órbita centro de masa del harnero con y sin carga, (b) Comparación del modelo lineal y no lineal en el espectro logarítmico de la respuesta vertical en $A$. es igual a $182^{\circ}$ la órbita se asemeja a una recta, que es lo que se observa en la Figura 3 (a). La dirección de las órbitas $\gamma=139\left[^{\circ}\right]$ es coincidente con la dirección de la fuerza más la inclinación del harnero $\alpha+\beta=139,8\left[^{\circ}\right]$, con una diferencia debido al movimiento de este.

Para este harnero simulado, por ser la respuesta angular de baja amplitud, $\Theta=3 \cdot 10^{-3}\left[{ }^{\circ}\right]$, la no linealidad geométrica no se hace presente, por lo que la diferencia entre el modelo lineal y no lineal es despreciable en el dominio tiempo, pero sí es evidente en el espectro en frecuencias. El no lineal en el centro de masa contiene, además de la componente a $1 \mathrm{X}$, una $2 \mathrm{X}$ de baja amplitud e inherente a su funcionamiento, como el otro es lineal, solo responde a la frecuencia de excitación (no aparece la 2X). Sin embargo, al calcular en los apoyos, al trasladar y rotar los ejes, aparece la componente $2 \mathrm{X}$ en el lineal, pero de menor amplitud a la calculada con el otro (100 veces menor). La Figura 3 (b) presenta el espectro de la respuesta vertical en $A$ de los dos modelos, que para ambos existe una componente $2 \mathrm{X}$.

\section{Simulaciones con cambio en la rigidez}

Se realizan simulaciones del sistema con carga variando la rigidez discretamente en uno de los apoyos, primero $A$ y luego en $B$. Los cambios de la rigidez se llevan a cabo desde $100 \%$ a $20 \%$, para luego obtener las respuestas estacionarias.

En la respuesta en dominio tiempo y frecuencia las diferencias son mínimas, mas en las órbitas (representación gráfica de la trayectoria de un punto) se observa un cambio de dirección $\gamma$ (ángulo de tiro de la órbita), por la disminución de rigidez. Como los harneros vibratorios cuentan con acelerómetros en los apoyos, los resultados de estas simulaciones son útiles para comparar con mediciones en terreno.

El cambio de dirección comentado, es expuesto en la Figura 4 (a), para los puntos $A, B$ y centro de masa $(\mathrm{cm})$, para distintos porcentajes de $K_{a}$ y (b) muestra su cambio relativo a la posición inicial. Se concluye que ante un cambio de rigidez en $A$ las direcciones de las órbitas varían en igual cantidad $\Delta \gamma$ a lo largo de todo el harnero, demostrado por los cálculos efectuados en los tres puntos. 
De igual forma al cambio en A, se modifica en porcentaje $K_{b}$, para calcular con el mismo procedimiento. La Figura 5 (a) muestra el ángulo de tiro del punto $A, B \mathrm{y} \mathrm{cm}$ para los porcentaje de $K_{b}$ y (b) el cambio de este. Análogamente al cambio de rigidez en $A$, se destaca que ante un cambio de rigidez en $B$ la dirección de cada órbita varía la misma cantidad a lo largo de todo el harnero.

Este cambio de dirección en las órbitas es importante porque simboliza la variación en la inclinación del harnero que, como se ha dicho, afecta en su rendimiento. También esto es usado por los fabricantes para determinar un rango óptimo de operación de manera de asegurar una clasificación de mineral adecuada [9].

\section{Cambio en la frecuencia natural}

Los harneros vibratorios utilizados en la minería trabajan sobre su primera frecuencia natural, por ende, en una partida o detención deben pasar por ella y entrar en resonancia. Como la frecuencia (a)
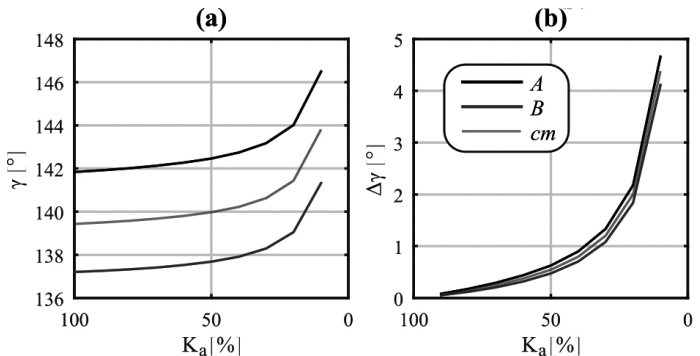

Figura 4. Ángulo de tiro $\gamma$ versus cambio de en la rigidez en A: (a) Ángulo de tiro, (b) Cambio en el ángulo de tiro con respecto al $100 \%$ de la rigidez en $A$. (a)

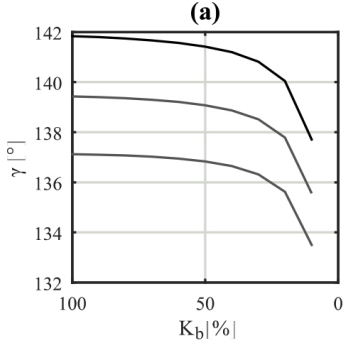

(b)

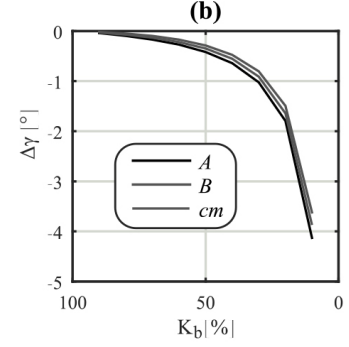

Figura 5. Ángulo de tiro $\gamma$ versus rigidez en $B$ : (a) Ángulo de tiro, (b) Cambio en el ángulo de tiro con respecto al $100 \%$ de la rigidez en $B$. natural depende de la rigidez, se puede buscar cambios en la frecuencia natural y relacionarlos con la disminución de rigidez. Para ello se simulan partidas del harnero sin carga y mediante un espectro de la respuesta vertical se busca la frecuencia de la máxima componente del espectro, para comparar las respuestas del centro de masa, apoyos y la frecuencia natural del sistema lineal, que equivale a $\omega_{n} \sqrt{k / m}$ y $\omega_{d}=\omega_{n} \sqrt{1-\xi_{2}}$.

El tiempo de la simulación son 100 [s] para tener $\Delta f=0,01[\mathrm{~Hz}]$ y aproximando a un número entero de ciclos del $\omega_{d}$ del modelo lineal ya calculado. $\mathrm{La}$ velocidad del sistema de excitación es simulada con una función seno que varía crecientemente desde 0 a 98,96 [rad/s]. Los resultados de las frecuencias son mostrados en la Figura 6, donde cada una representa la frecuencia encontrada en dicho punto, con respecto a la rigidez en $A$ (abscisa) y $B$ (ordenada).

Los resultados se ven afectados por la tasa de la partida simulada, como es observado en [10], cambiando la magnitud de la frecuencia encontrada.

La Figura 6 (a) y (b) presenta las frecuencias naturales del centro de masa del modelo lineal y la velocidad crítica del no lineal respectivamente. Los valores desde el centro hacia el extremo superior derecho
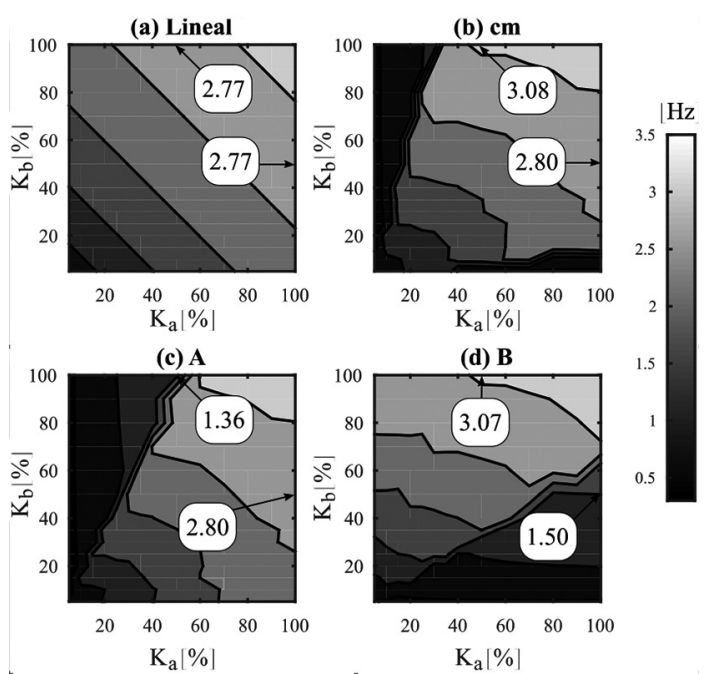

Figura 6. (a) Frecuencia natural amortiguada del sistema lineal equivalente. Frecuencia de la máxima componente espectral en: (b) El centro de masa, (c) Apoyo A, (d) Apoyo B. 
de la figura (sin pérdida de rigidez) no exhiben grandes diferencias, pero para $K_{a}<20 \%$ y $K_{b}<30 \%$, se acentúa la discrepancia por la no linealidad, ya que para esos valores la frecuencia de la máxima componente no corresponde a la frecuencia natural en $y$, sino que a la de $\theta, x$ o un modo que presenta la combinación de ambos ( $x$ y $\theta)$.

En los apoyos hay mayor influencia en el cambio de la frecuencia de la máxima componente con la variación de la rigidez en el mismo punto, que se destaca cuando la rigidez es menor a su mitad. Por ejemplo, con los valores mostrados en la Figura 6 (c) y (d), se observa que para $100 \%$ $K_{a}$ y $50 \% K_{b}$, en $A$ la diferencia es nula y en B es de $46 \%$ con respecto al cm, para $50 \% K_{a}$ y $100 \% K_{b}$, en $A$ existe un $56 \%$ de diferencia y en $B$ cercana a cero.

\section{CONCLUSIONES}

Los modelos dinámicos vistos en la introducción son lineales, por lo que no son aptos de usar cuando la rotación es alta. Además presentan errores en su desarrollo y faltan simulaciones que permitan conocer condiciones distintas a las normales de su función.

Se desarrolló un nuevo modelo dinámico no lineal capaz de describir el funcionamiento de un harnero y se realizan distintas simulaciones: con carga, sin carga, variando la rigidez de los apoyos y una partida.

Al comparar el modelo lineal con el no lineal la respuesta en condiciones nominales es muy similar, pues la respuesta angular es de baja amplitud. Por el traslado y rotación de ejes a los apoyos, el modelo lineal también responde con una componente $2 \mathrm{X}$.

Se destaca que ante una disminución en la rigidez de los apoyos las órbitas del harnero responden igualmente, cambiando la misma cantidad el ángulo de tiro. Esto es obtenido para el caso en 2D, pero se puede extrapolar para el caso en 3D, de esta forma si un apoyo pierde rigidez se puede saber cuál es al medir las órbitas en los cuatro apoyos.

El harnero al trabajar sobre su frecuencia natural debe entrar en resonancia y en el espectro se puede detectar cambios en su frecuencia natural por la pérdida de rigidez. Se debe notar que a baja rigidez la frecuencia de las máximas componentes espectrales son las correspondientes a $x$ y $\theta$, por lo que se deben analizar todos los peaks para detectar los cambios de rigidez.

Con los resultados de las órbitas en estado estacionario y espectro en una partida o detención, es posible evaluar el estado de los resortes con el fin de asegurar la alta eficiencia requerida y, con conocimiento de su condición, planificar el reemplazo de los resortes.

\section{REFERENCIAS}

[1] C. Liu, H. Wang, Y. Zhao, L. Zhao and H. Dong. "DEM simulation of particle flow on a single deck banana screen". International Journal of Mining Science and Technology. Vol. $23 \mathrm{~N}^{\mathrm{o}} 2$, pp. 273277. 2013.

[2] L. Zhao, Y. Zhao, C. Liu, J. Li and H. Dong. "Simulation of the screening process on a circularly vibrating screen using 3D-DEM". Mining Science and Technology. Vol. 21 No 5, pp. 677-680. 2011.

[3] X.M. He and C.S. Liu. "Dynamics and screening characteristics of a vibrating screen with variable elliptical trace". Mining Science and Technology. Vol. 19 No 4, pp. 508-513. 2009.

[4] C. Liu, L. Peng and F. Li. "Survey of signal processing methods and research on vibrating screen fault diagnosis". Mechanic Automation and Control Engineering. Mongolia Interior, China. Julio 2011.

[5] B.A. Wills and T. Napier-munn. "Mineral Processing Technology:An Introduction to the Practical Aspects of Ore Treatment and Mineral Recovery". ButterworthHeinemann. Séptima Edición. 2006. ISBN: 978-0-7506-4450-1.

[6] V.K. Karra. "Development of a Model for Predicting the Screening Performance of a Vibrating Screen". CIM Bulletin. Vol. 72 No 804, pp. 167-171. 1979.

[7] L. Peng, C. Liu, J. Li and H. Wang. "Staticdeformation based fault diagnosis for damping spring of large vibrating screen". Journal 
of Central South University. Vol. $21 \mathrm{~N}^{\circ} 4$, pp. 1313-1321. 2014.

[8] C.B. Moler. "Numerical Computing with MATLAB". Society for Industrial and Applied Mathematics, pp. 185-234. 2004.

[9] M.I.I. Metso. "ScreenWatch ${ }^{\circledR}$ continuous monitoring system: Protecting the operatio- nal integrity of Metso's entire Opti-Flo® range". 2014.

[10] S.-M. Wang. Q.-S. Lu, Q. Wang and P. Xu. "Reducing the amplitude of vibration at resonances by phase modulation". Journal of Sound and Vibration. Vol. $290 \mathrm{~N}^{\circ} 1-2$, pp. 410-424. Febrero 2006. 